\title{
BOUNDEDNESS PROPERTIES FOR LINEAR ORDINARY DIFFERENTIAL EQUATIONS
}

\author{
DAVID LOWELL LOVELADY
}

ABSTRACT. In comparing a linear equation and the associated nonhomogeneous equation, it is shown that if every bounded forcing function and every $\mathscr{L}^{1}$ forcing function yields at least one bounded solution, then the bounded subset of the solution family of the homogeneous equation is uniformly ultimately bounded.

I. Introduction and results. Let $Y$ be a finite dimensional normed linear space with norm || , and let $R^{+}$be the set of all nonnegative real numbers. Let $\mathscr{A}$ be the algebra of linear functions from $Y$ to $Y$ with induced norm \|\| . We propose to compare various boundedness properties of the solutions of the problem

$$
v^{\prime}(t)=A(t) v(t),
$$

where $A$ is a continuous function from $R^{+}$to $\mathscr{A}$. We shall also be concerned with the associated problem

$$
u^{\prime}(t)=f(t)+A(t) u(t),
$$

where $f$ is always at least locally integrable.

T. Yoshizawa [7], S. R. Bernfeld [1], and others have studied the properties of uniform boundedness and uniform ultimate boundedness for (1), primarily in connection with trying to preserve these properties under perturbations. On the other hand, W. A. Coppel [3], [4], R. Conti [2], V. A. Staikos [5], and P. Talpalaru [6] have studied the stability and boundedness properties inherited by (1) when one requires that each member $f$ of various function spaces yield a bounded solution $u$ of (2). Little is known about the connections between these two schools of thought. An exception: Coppel [4, Theorem 1, p. 131] has shown that uniform boundedness for (1) (see [1, Definition 2.1]) is equivalent to the requirement that if $f$ is in $\mathscr{L}^{1}\left[R^{+}, Y\right]$ then every solution $u$ of (2) is bounded. We shall obtain further connections.

We shall say that (1) is quasi-uniformly-bounded (QUB) if and only if whenever $B$ is a bounded subset of $Y$ there is a number $\mu$ such that if $c$ is

Received by the editors November 9, 1972 and, in revised form, February 14, 1973. AMS (MOS) subject classifications (1970). Primary 34A30, 34D35; Secondary 34C99.

Key words and phrases. Uniform boundedness, uniform ultimate boundedness, evolution systems. 
in $R^{+}$, if $v$ solves (1) on $[c, \infty)$, if $v(c)$ is in $B$, and if $v$ is bounded, then $|v(t)| \leqq \mu$ whenever $t$ is in $[c, \infty)$. Note that QUB does not require that all solutions of (1) be bounded; it requires that the family of all bounded solutions be uniformly bounded. We shall say that (1) is quasi-uniformlyultimately-bounded (QUUB) if and only if there is a number $\lambda$ such that if $B$ is a bounded subset of $Y$ then there is a positive number $\tau$ such that if $c$ is in $R^{+}$, if $v$ solves (1) on [c,, ), if $v(c)$ is in $B$, and if $v$ is bounded, then $|v(t)| \leqq \lambda$ whenever $t$ is in $(c+\tau, \infty)$. The relationship between QUUB and uniform ultimate boundedness is analogous to that between QUB and uniform boundedness. To see that these "quasi" properties are realistic, consider the very simple system

$$
x_{1}^{\prime}(t)=-x_{1}(t), \quad x_{2}^{\prime}(t)=x_{2}(t) .
$$

A solution of this system is bounded if and only if $x_{2}=0$. Yet the set of all solutions with $x_{2}=0$ is both uniformly bounded and uniformly ultimately bounded. Thus the system satisfies QUB and QUUB but still has some unbounded solutions.

The following theorem is our main result.

THEOREM 1. Suppose that (1) is $Q U B$ and that if $f$ is a bounded continuous function from $R^{+}$to $Y$ then there is at least one bounded solution $u$ of (2) on $R^{+}$. Then (1) is QUUB.

Note what Theorem 1 says in the case that every solution of (1) on $R^{+}$is bounded. In this case QUB and QUUB reduce to uniform boundedness and uniform ultimate boundedness respectively.

II. Proofs. We shall first prove a more general theorem and then show how Theorem 1 follows as a consequence. Let $\mathscr{B}$ be a (real or complex) Banach algebra (with or without identity) with norm \|\| , and let $D=$ $\{(t, s): 0 \leqq s \leqq t\}$.

THEOREM 2. Let $E$ be a continuous function from $D$ to $\mathscr{B}$ and suppose that if each of $(t, s)$ and $(s, r)$ is in $D$ then $\|E(t, s)\|>0$ and $E(t, s) E(s, r)=$ $E(t, r)$. Suppose further that $K$ is a positive number, that

$$
\int_{0}^{t}\|E(t, s)\| d s \leqq K
$$

whenever $t$ is in $R^{+}$, and that

$$
\|E(t, s)\| \leqq K
$$

whenever $(t, s)$ is in $D$. Then

$$
\lim _{c \rightarrow \infty} \sup \{\|E(t, s)\|: t-s \geqq c\}=0 .
$$


Proof. Let $k=1 / K$. We shall actually show that if $(t, s)$ is in $D$ and $t-s \geqq 1$ then

$$
\|E(t, s)\| \leqq K^{2} e^{k} e^{k(s-t)} .
$$

Clearly (5) follows from (6).

Let $s$ be in $R^{+}$, and let $\phi$ be given on $[s, \infty)$ by $\phi(t)=\|E(t, s)\|^{-1}$. Now, from (4), $\int_{s}^{s+1} \phi(t) d t \geqq k$. Also, if $t$ is in $[c, \infty)$,

$$
\begin{aligned}
\phi(t)^{-1} \int_{s}^{t} \phi(r) d r & =\left\|\left(\int_{s}^{t} \phi(r) d r\right) E(t, s)\right\| \\
& =\left\|\int_{s}^{t} \phi(r) E(t, r) E(r, s) d r\right\| \\
& \leqq \int_{s}^{t} \phi(r)\|E(t, r) E(r, s)\| d r \\
& \leqq \int_{s}^{t}\|E(t, r)\| d r \leqq K,
\end{aligned}
$$

so $\int_{s}^{t} \phi(r) d r \leqq K \phi(t)$. Thus, if $t \geqq s+1$,

and

$$
K \phi(t) \geqq \int_{s}^{s+1} \phi(r) d r+\int_{s+1}^{t} \phi(r) d r \geqq k+\int_{s+1}^{t} \phi(r) d r,
$$

Thus, if $t \geqq s+1$,

$$
\phi(t) \geqq k^{2}+k \int_{s+1}^{t} \phi(r) d r
$$

$$
\phi(t) \geqq k^{2} e^{k(t-s-1)}=k^{2} e^{-k} e^{k(t-s)} \quad \text { so } \quad\|E(t, s)\|=\phi(t)^{-1} \leqq k^{2} e^{k} e^{k(s-t)} .
$$

This completes the proof of Theorem 2 .

Let $M$ be that linear subspace of $Y$ to which $x$ belongs only in case the solution $v$ of (1) such that $v(0)=x$ is bounded. Let $P$ in $\mathscr{A}$ be a projection with range $M$. If $P=0$, then QUB and QUUB are both satisfied trivially, so we shall assume $P \neq 0$. Let $\Phi$ be the "fundamental solution" for (1), i.e., $\Phi$ is that continuously differentiable function from $R^{+}$to $\mathscr{A}$ such that $\Phi(0)$ is the identity and $\Phi^{\prime}(t)=A(t) \Phi(t)$ whenever $t$ is in $(0, \infty)$. Recall that each value of $\Phi$ is invertible.

Now suppose that $c$ is in $R^{+}, v$ solves (1) on $[c, \infty), v$ is bounded, and $v(c)=x$. We can extend $v$ to a solution of $(1)$ on $R^{+}$, and this extension is, of course, bounded. Now $v(t)=\Phi(t) \Phi(c)^{-1} x$ whenever $t$ is in $R^{+}$, so $v(0)=\Phi(c)^{-1} x$. Since $v$ is bounded, $v(0)$ is in $M$, so $P \Phi(c)^{-1} x=\Phi(c)^{-1} x$, and we see that $v(t)=\Phi(t) P \Phi(c)^{-1} x$ whenever $t$ is in $R^{+}$and, in particular, whenever $t$ is in $[c, \infty)$. On the other hand, if $y$ is in $Y$ and $w$ is given on 
$[c, \infty)$ by $w(t)=\Phi(t) P \Phi(c)^{-1} y$, then $w$ is a bounded solution of $(1)$ on $[c, \infty)$.

These observations make it clear that QUB is equivalent to requiring that

$$
\sup \left\{\left\|\Phi(t) P \Phi(s)^{-1}\right\|:(t, s) \text { is in } D\right\}
$$

be finite and that QUUB is equivalent to

$$
\lim _{c \rightarrow \infty} \sup \left\{\left\|\Phi(t) P \Phi(s)^{-1}\right\|: t-s \geqq c\right\}=0 .
$$

Also, Coppel [4, Theorem 1, p. 131] has shown that the second hypothesis of Theorem 1 implies that there is a number $K$ such that $\int_{0}^{t}\left\|\Phi(t) P \Phi(s)^{-1}\right\| \leqq$ $K$ whenever $t$ is in $R^{+}$. Thus, if we let $\mathscr{B}=\mathscr{A}$ and let $E$ be given by $E(t, s)=\Phi(t) P \Phi(s)^{-1}$, then the hypotheses of Theorem 1 tell us that (3) and (4) follow. Hence (5) is true, and Theorem 1 is proved.

It follows from [4, Theorem 2, p. 131] and the above computations that if whenever $f$ is in $\mathscr{L}^{1}\left[R^{+}, Y\right]$ there is a bounded solution $u$ of (2) on $R^{+}$, then (1) is QUB. Thus we see from Theorem 1 that if every bounded continuous $f$ and every $f$ in $\mathscr{L}^{1}\left[R^{+}, Y\right]$ yields a bounded solution $u$ of (2) then the bounded subset of the solutions of (1) is uniformly ultimately bounded.

\section{REFERENCES}

1. S. R. Bernfeld, Perturbing uniform ultimate bounded differential systems, SIAM J. Math. Anal. 3 (1972), 358-370.

2. R. Conti, On the boundedness of solutions of ordinary differential equations, Funkcial. Ekvac. 9 (1966), 23-26. MR 37 \#3102.

3. W. A. Coppel, On the stability of ordinary differential equations, J. London Math. Soc. 39 (1964), 255-260. MR 29 \#1393.

4. - - Stability and asymptotic behaviour of differential equations, Heath, Boston, Mass., 1965. MR 32 \#7875.

5. V. A. Staikos, $A$ note on the boundedness of solutions of ordinary differential equations, Boll. Un. Mat. Ital. (4) 1 (1968), 256-261. MR 37 \#1704.

6. P. Talpalaru, Quelques problèmes concernant l'équivalence asymptotique des systèmes différentiels, Boll. Un. Mat. Ital. (4) 4 (1971), 164-186.

7. T. Yoshizawa, Stability theory by Liapunov's second method, Publ. Math. Soc. Japan, no. 9, Math. Soc. Japan, Tokyo, 1966. MR 34 \#7896.

Department of Mathematics, Florida State University, Tallahassee, Florida 32306 